\title{
Guerra e prigionia. Insegnare la Prima guerra mondiale nelle scuole secondarie di secondo grado
}

\author{
di Giuseppe Ferraro
}

Nov 4, 2020 | Didattica in classe, $\underline{\text { In evidenza }}|\underline{0}|$

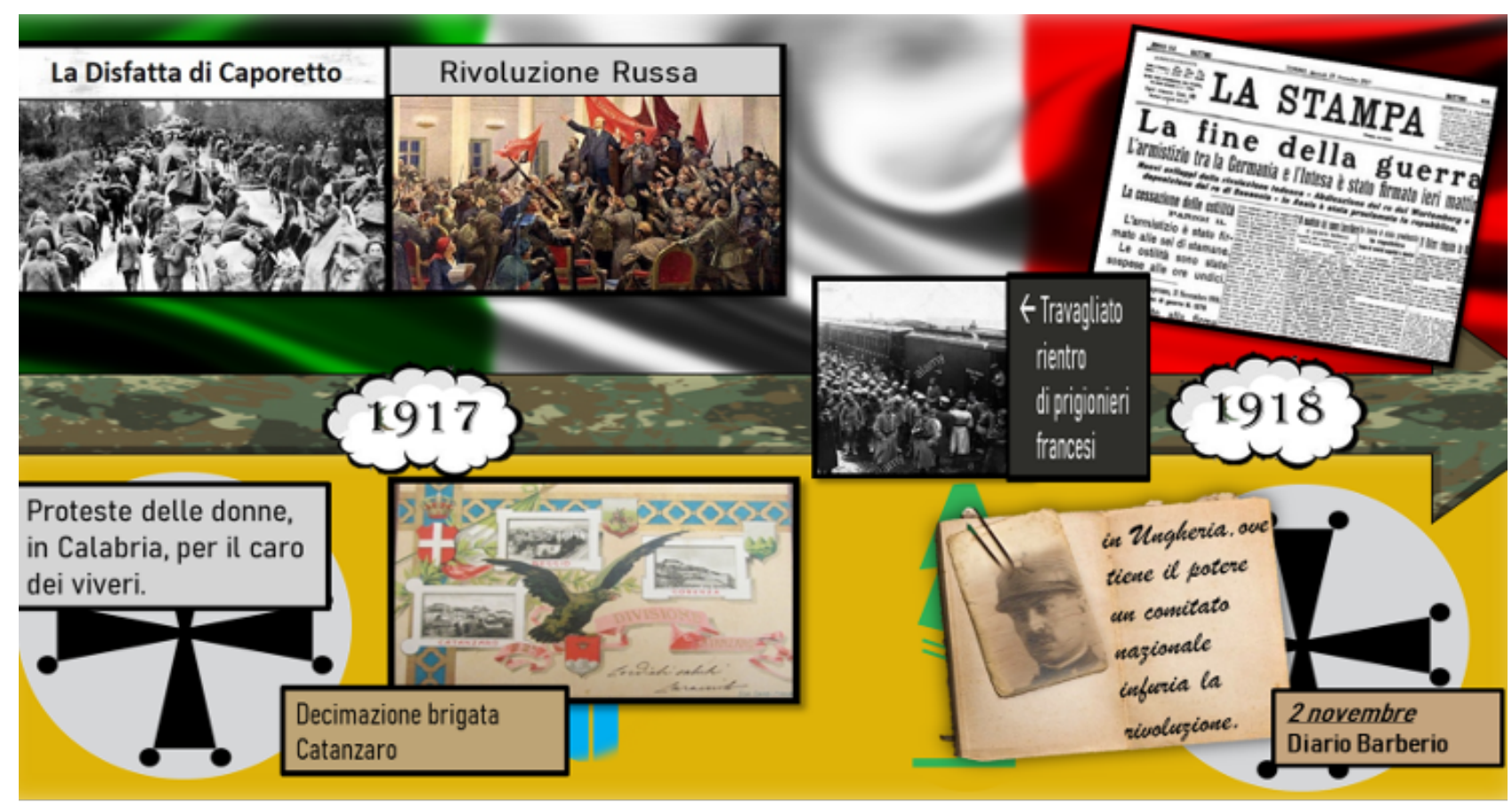

\section{Abstract}

Nell'ultimo anno delle scuole secondarie di secondo grado, il tema della Prima guerra mondiale può diventare un terreno abbastanza accidentato per la complessità e la ricchezza dei contenuti, oppure una risorsa sia per i docenti che per i gruppi classe, anche in relazione alla didattica a distanza. In questo articolo si è pensato di offrire ai docenti alcuni suggerimenti e pratiche su come rendere la lezione frontale su tale argomento più efficace, curiosa, funzionale a valorizzare le attività laboratoriali e, in caso della didattica a distanza, le ore asincrone[1].

Le considerazioni contenute in questo articolo nascono dalle esperienze svolte in molte scuole secondarie, promosse dalla sezione didattica dell'Istituto Calabrese per la Storia dell'Antifascismo e dell'Italia Contemporanea, e sono state oggetto di una relazione, esposta dall'autore nell'ambito del Terzo seminario nazionale SISSCO di didattica della storia (Siracusa, 12-13 aprile 2019)[2].

\section{Premessa}

Questo tipo di lezione frontale è pensata con un approccio tematico orientato a valorizzare gli aspetti socio-culturali della Prima guerra mondiale: il tema scelto è quello della prigionia, che sembra suscitare un maggiore interesse negli studenti e anche facilitare attività laboratoriali originali che permettano di far acquisire ai gruppi classe sia riferimenti metodologici, propri della disciplina, che un approccio multidisciplinare. Da non trascurare, inoltre, la possibilità di approfondire, attraverso le vicende del primo conflitto mondiale, anche temi relativi all'educazione 
civica. L'impostazione di una lezione sulla Prima guerra mondiale, che valorizzi un continuo dialogo tra passato e presente, motiva ancora di più i ragazzi a studiare l'argomento, a comprendere e problematizzare l'evento come qualcosa che ancora bussa all'uscio di casa propria. È molto importante infatti interrogare il passato sui problemi, le complessità o le esperienze del presente[3], facendo capire agli studenti come la storia ha a che fare con la loro vita, con la società in cui vivono e con la loro comprensione generale dell'esistenza umana[4]. Bisogna però prestare attenzione ad un rischio: evitare di schiacciare la storia su una sorta di "presentismo"[5] che porterebbe ad un appiattimento degli orizzonti[6]. Non si può indagare il passato con chiavi di lettura e categorie mentali attuali[7].

\section{Una lezione frontale "rivisitata" e diversificata}

La lezione riguarda la prigionia durante la Prima guerra mondiale e ha il seguente titolo: Prigionieri della guerra. Il fine è utilizzare l'argomento della prigionia, reso il più possibile accattivante dal docente, come cavallo di Troia per addentrarsi con maggiore curiosità e passione nello studio della Prima guerra mondiale, dei suoi effetti e riflessi, anche attuali. Una lezione strutturata per una durata di circa 20 minuti, con l'intenzione di rendere le conoscenze dinamiche, proiettate a diventare competenze agite. Tempi brevi, ma efficaci, con lo scopo di valorizzare anche la partecipazione del gruppo classe, non rendere l'attività unilaterale e frontale. Il docente non ha nemmeno l'obiettivo di esaurire, completare e terminare il percorso didattico, ma quello di lasciare volutamente dei "non finiti", dove confrontarsi per riempirli o lasciarli al lavoro autonomo della classe. La sua diversificazione con oggetti, immagini, carte geografiche, linee del tempo, audio e parole chiave, mira a valorizzare nella didattica stimoli cognitivi, visivi ed emozionali.

\section{Valorizzare il tempo}

Come fare in poco tempo a rendere un'attività didattica creativa ed efficace? I docenti di storia nella secondaria di secondo grado (escluso l'indirizzo liceo classico con tre ore) hanno infatti a disposizione solo due ore settimanali, che saranno molte di meno, tra verifiche e varie problematiche interne ad ogni scuola che sottraggono sempre di più tempo alla lezione in aula. La lezione viene per questo calibrata intorno ai 20 minuti, prendendo in considerazione una scuola dove lo spezzone orario è di 60 minuti; i successivi 20 minuti servono alla discussione critica sul tema trattato. L'altra frazione di tempo è impiegata per pianificare e affidare le consegne in una dimensione laboratoriale.

In queste tappe il docente dovrà creare condizioni anche a livello sociale e psicologico, non solo culturale e didattico, per costruire l'autonomia di lavoro degli studenti: «la competenza delle competenze»[8]. La didattica a distanza inoltre ha evidenziato come la concentrazione, l'interesse e l'empatia verso la lezione o una qualsiasi attività didattica sia minore rispetto al lavoro in aula. Una lezione diversificata potrebbe aiutare notevolmente il docente nel migliorare, calibrare e gestire l'attenzione del gruppo classe.

\section{Le fasi}

La lezione è articolata in 5 slide. Le slide contengono una cronologia o una linea del tempo; mappe geografiche; una sequenza numerica che in base all'argomento può essere un grafico con dati 
economici, demografici o di altra natura; fonti iconografiche e grafiche; 4 parole chiave su cui basare le proprie argomentazioni durante la conclusione. Il docente presenta anche tre oggetti o elementi relativi al tema, che hanno lo scopo di "rompere il ghiaccio" a inizio lezione con una fase di brainstorming, oppure, dopo, suscitare il dibattito.

Questi sono gli oggetti scelti:

1. Un pallone utilizzato nei campi di prigionia

2. Una banconota in circolazione nei campi di prigionia (in questo caso a Dunaszerdahely allora in territorio ungherese)

3. Un cane, mascotte di un reggimento

La fase di brainstorming iniziale permette di sollecitare l'attenzione del gruppo classe sul perché siano stati scelti questi elementi e su quali funzioni possano avere avuto nei campi di prigionia. Durante la discussione è necessario allargare l'orizzonte sui temi della cittadinanza.

Il pallone non rappresentava solo un utile diversivo sociale nelle lunghe e monotone giornate dei campi di prigionia, ma era anche un simbolo di come l'Europa si fosse costruita e conosciuta proprio nell'ambito di tragedie nazionali e umane, in esperienze tristissime come la prigionia (il confino, l'internamento e le deportazioni). Il gioco aveva permesso spesso incontri sia tra connazionali, provenienti da varie regioni, che tra prigionieri di nazionalità diversa. In alcuni casi infatti le autorità austro-ungariche consentirono ai prigionieri stranieri tra loro, divisi nei vari settori del campo, di interagire per disputare iniziative di questo tenore.

Nel caso della banconota, invece, la riflessione permette di analizzare questioni relative alla vita economica e sociale della comunità di prigionia, spaziando sul ruolo delle convenzioni internazionali nella difesa e tutela dei diritti nei campi di prigionia e sulla loro funzione contemporanea.

Per quanto riguarda il cane, può emergere come la guerra totale avesse coinvolto anche animali e ambiente naturale. Gli animali nei campi, come il gioco, avevano una funzione sociale importante: distrarre e confortare i prigionieri. In molti campi, come in quello ad esempio di Dunaszerdahely, venne fondata, ad opera dei prigionieri, un'associazione in difesa e protezione degli animali del campo o di quelli che venivano abbandonati a causa della morte o del trasferimento del proprietario. Anche questo tema tocca evidenti aspetti relativi alla cittadinanza attiva: è possibile infatti, consegnare ad alunni coinvolti in esperienze di volontariato in difesa degli animali il compito di approfondire, attraverso la metodologia della classe capovolta, il ruolo degli animali in guerra. Conoscere gli hobby, le passioni, gli interessi dei membri del gruppo classe può essere funzionale a valorizzarli all'interno delle attività didattiche.

I tre oggetti vengono presentati con un breve sottotitolo: Tre oggetti tante storie. Questo titolo serve a sottolineare come ogni esperienza storica venga vissuta, subita, gestita e assimilata in maniera diversa dai soggetti interessati. Quei tre oggetti-elementi nella percezione degli osservatori di oggi, possono avere vari significati e originare più varianti del racconto corale della Grande guerra.

\section{Il tempo in tempi}

La prima slide della lezione è pensata per contestualizzare cronologicamente il tema della prigionia e della Prima guerra mondiale con la presentazione di una linea del tempo, che includa le coordinate 
generali di inizio e fine del conflitto e i periodi di maggiore aumento della prigionia. Il 1917 è stato infatti evidenziato per i fatti di Caporetto e l'aumento dei prigionieri italiani nei campi austroungarici e tedeschi. Vengono evidenziate alcune date con un numero maggiore o minore di cerchi concentrici: quindi soprattutto in relazione al 1917-1918 per quanto riguarda la questione dei prigionieri di guerra italiani.

Anche in questo caso può essere affidata, dopo lo svolgimento della lezione, una consegna alla classe: realizzare la loro linea del tempo (anche in gruppo), che dovrà però avere un doppio registro: sulla parte superiore indicare eventi relativi al contesto nazionale e internazionale, in quella inferiore la storia del territorio anche in base alle micro ricerche affidategli in altri momenti e alle "esperienze vicine": un bisnonno morto in un campo di prigionia oppure una vicenda relativa alla storia locale. In questo caso la linea del tempo non presenta la storia come qualcosa di estraneo e lontano dai propri vissuti. Il docente, inoltre, dovrebbe spingere il gruppo classe ad interrogarsi sul tempo, la sua linearità, ma anche profondità, durata e diversità di percezione da parte degli individui. La percezione che ha del tempo un soldato in trincea durante la Prima guerra mondiale è molto diversa da quella di un suo simile nei campi di prigionia.

\section{Il tempo in tempi}

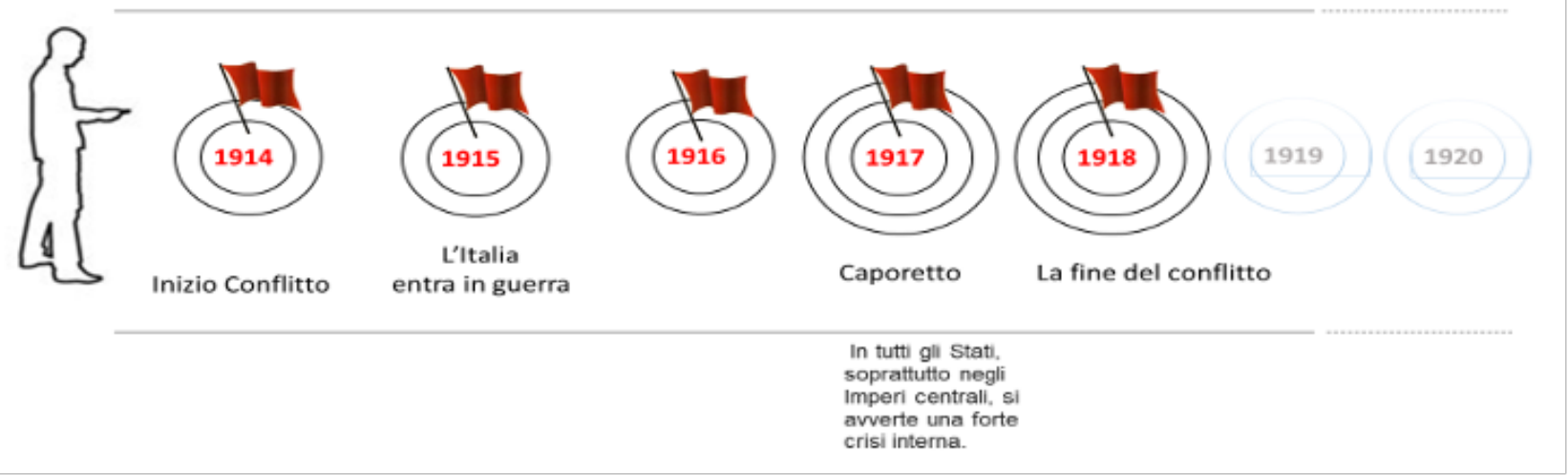

Riportiamo come esempio quanto realizzato dalla classe V A, in particolare dagli alunni Quintino Berardi e Pietro De Simone, del Polo liceale di Rossano (CS), a cui è stato proposto il laboratorio nell'anno scolastico 2019-20. Questi studenti hanno realizzato la linea del tempo inserendo alcuni eventi relativi al quadro nazionale-internazionale, come la Rivoluzione russa, Caporetto e i prigionieri francesi, ma anche questioni relative a precedenti esperienze di laboratorio sul territorio o scaturite durante il dibattito in aula. Nel registro in basso infatti hanno segnalato conoscenze acquisite durante le precedenti lezioni sulla Prima guerra mondiale dove si erano studiate fonti relativi alla storia locale. La linea del tempo in basso ha contestualizzato il conflitto in una situazione di forte instabilità per la Calabria, dovuta ai pesanti effetti del terremoto di Reggio e Messina del 1908. Sono state inserite la cartolina di un proprio parente al fronte e alcune notizie riguardanti l'ufficiale Bernardo Barberio di origine calabrese membro della Brigata Catanzaro, poi fatto anche prigioniero. 

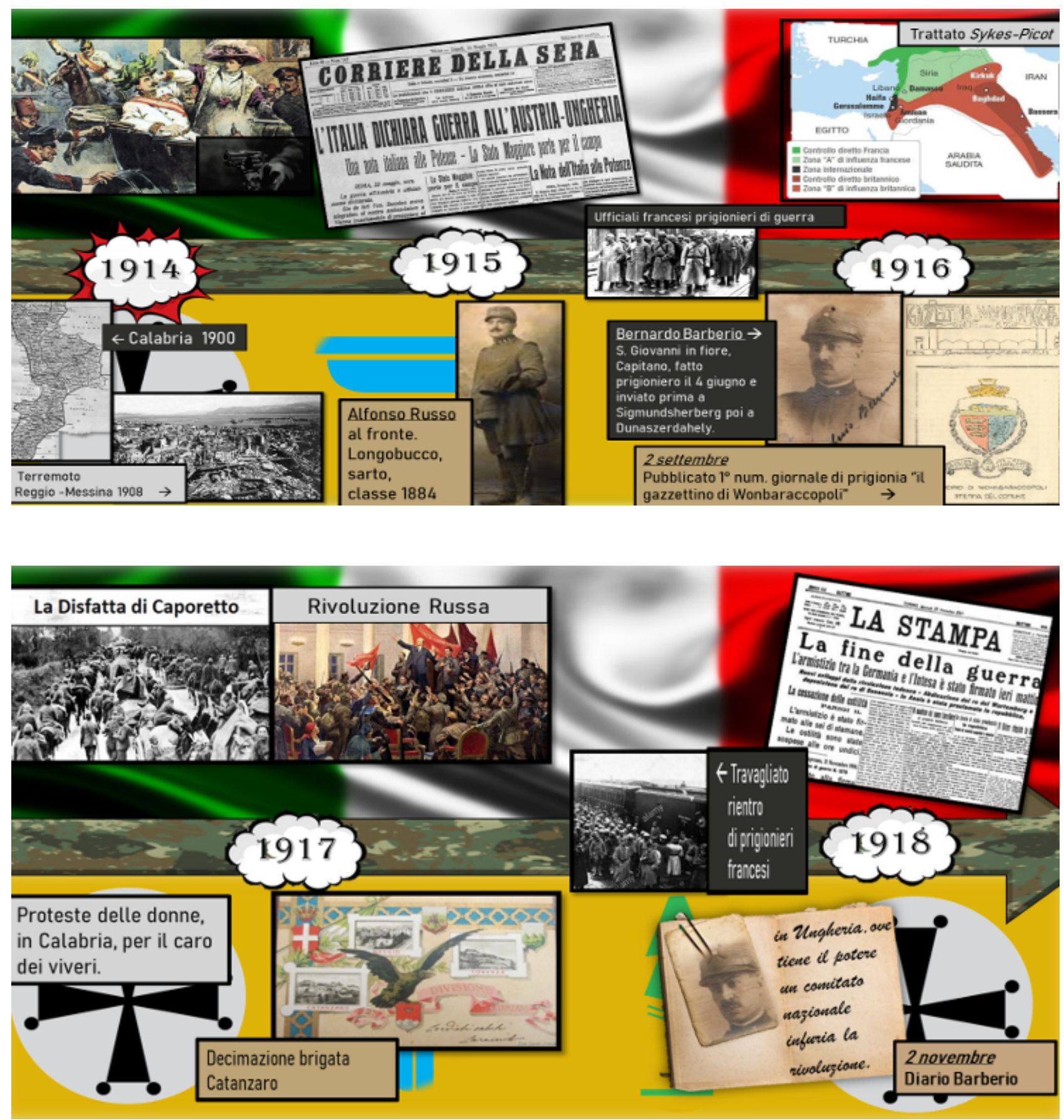

Carte che parlano, la geografia nelle ore di storia

Nella seconda slide della lezione vengono presentate alcune mappe con la dislocazione dei campi che ospitavano soprattutto militari italiani (C). Per le altre carte geografiche, sono state scelte quelle satiriche (A) in associazione a quelle politiche (B). Questa tecnica sembra suscitare maggiormente la curiosità e il processo di memorizzazione negli studenti, grazie alle figure che rappresentano le varie nazioni coinvolte. Le immagini satiriche sono state opportunamente inquadrate e commentate (Cosa ci vuole dire l'immagine? Chi è l'autore? Perché è stata realizzata e con quali scopi?). Devono essere infatti decodificate perché ben sappiamo che l'immagine non è sempre la verità e proprio da queste premesse il gruppo classe potrà acquisire anche competenze per la vita, ad 
esempio, essere osservatori preparati e capaci di interpretare la realtà e le informazioni in modo critico $^{[9]}$.
A) L'Europa tra nazionalismi e revanscismi
B) 1915, L'Europa in guerra (da https://storianet.blogspot.com)
C) 1916, I campi di prigiona austro-ungarici
D) 1916, I campi di prigionia tedeschi

\section{Il lavoro delle fonti}

La terza slide è dedicata alle fonti utili per studiare i campi di prigionia e la storia delle Prima guerra mondiale. Un'occasione per "sporcarsi un po' le mani”, ma soprattutto chiarire alcuni principi fondamentali del metodo storico, tenendo ben presente almeno quattro punti guida: selezionare, interrogare, interpretare e scrivere[10]. Si tratta di un momento formativo da richiamare per far comprendere come spesso l'uso distorto delle fonti possa produrre gravi danni ai fini della conoscenza storica (anche questo significa fare educazione civica, ogni giorno siamo sommersi da fake news).

In questa slide sono state presentate alcune delle fonti utili allo studio del tema trattato e delle indicazioni teoriche-metodologiche. È molto importante infatti proporre una dimensione teorica che consenta di orientarsi criticamente nella mole d'informazioni e narrazioni storiche presentata ogni giorno ad un pubblico sempre più vasto, soprattutto attraverso la rete[11]. Ma anche far comprendere al gruppo classe che una carta medievale, una miniatura, una cronaca, un'epigrafe si interrogano usando un determinato questionario e in maniera diversa rispetto ad una fonte giornalistica o ad un diario di un soldato della Prima guerra mondiale[12]. Trattare quindi in scala ridotta quelle tappe che gli studiosi compiono nella scelta, utilizzo e studio delle fonti[13]. Il docente dovrebbe il più possibile evidenziare che le fonti sono risorse dinamiche, forniscono informazioni diverse in base alle domande che gli vengono poste. In un certo senso si è sempre di fronte a qualcosa che in base a chi interroga, alla sua formazione, curiosità e attenzione, fornisce dati e risposte diverse ${ }^{[14]}$. Anche in questo caso può essere affidata una consegna: gli studenti, divisi in gruppi possono verificare se tra le proprie famiglie o sul territorio vi sono diari, corrispondenze o foto relative al primo conflitto mondiale. 


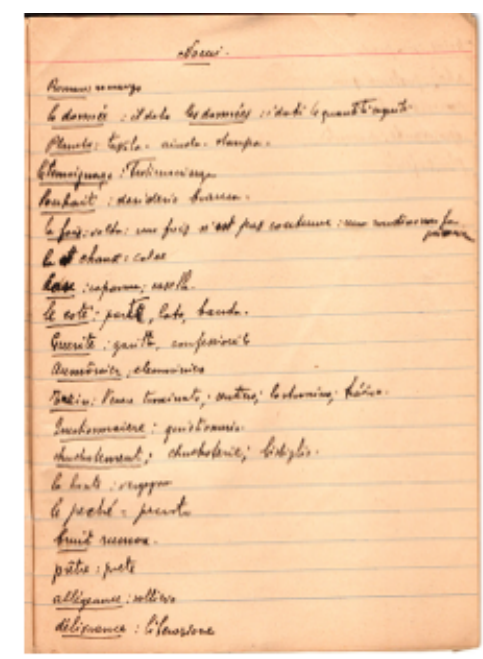

Piccolo vocabolario in un diario (1917)
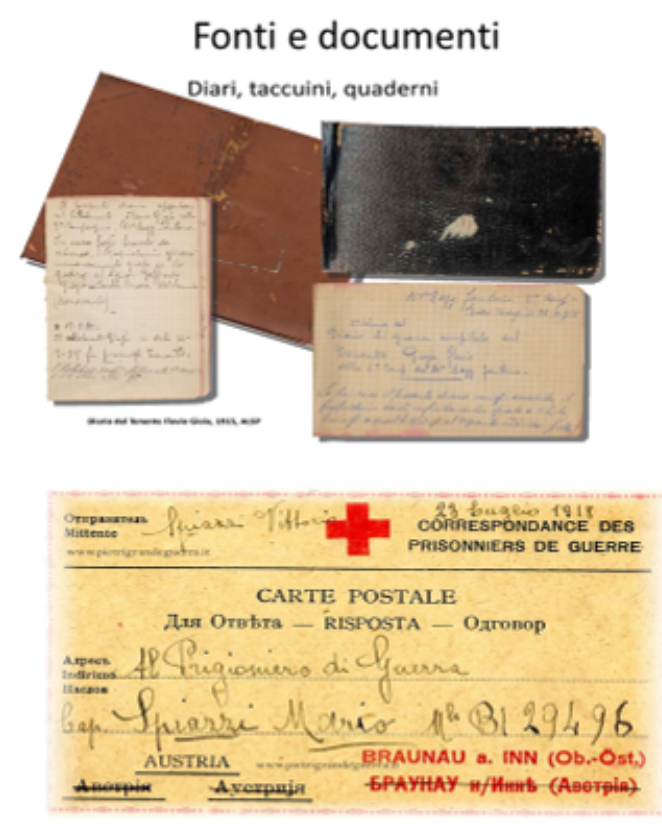

Lettere di prigionia

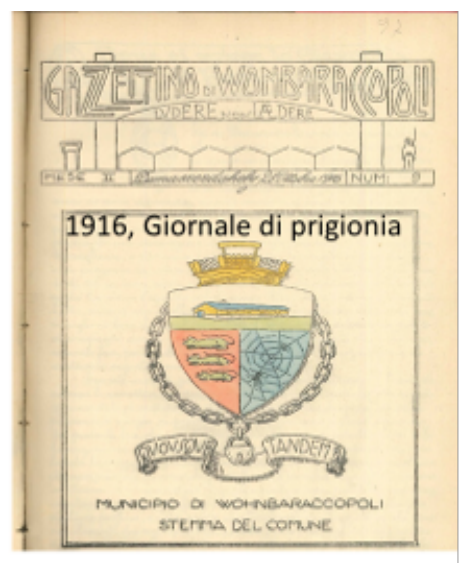

\section{Le persone, i dati e le statistiche}

Nella quarta slide viene presentata la dimensione quantitativa del fenomeno, associando foto e disegni della prigionia. Questo per sottolineare che dietro ogni numero vi sono persone, storie, esperienze sovrapponibili ma mai del tutto identiche, come la prigionia dimostra. La tabella dei morti e dei prigionieri permette di comparare il caso italiano con le altre realtà europee, quindi trasmettere con la lezione, anche in questo caso, una visione globale dell'evento, motivando meccanismi di apprendimento diacronico e sincronico. Le foto mostrano le condizioni di vita dei prigionieri nei campi, come venivano trattati, cosa facevano, quali erano davvero le loro condizioni fisiche e psicologiche. Anche per le foto è necessario specificare, per educare il gruppo classe alla problematicità, che non sempre ci dicono quello che immediatamente vediamo. 


\begin{tabular}{|c|c|c|c|}
\hline Nazione & $\begin{array}{c}\text { Morti causate dal } \\
\text { conflitto }\end{array}$ & Prigionieri & $\begin{array}{c}\text { Morti per causa di } \\
\text { prigionia }\end{array}$ \\
\hline Italia & 650.000 & 600.000 & 100.000 \\
\hline Francia & & 550.000 & 20.000 \\
\hline Inghilterra & & 180.000 & 12.000 \\
\hline Germania & & 700.000 & 35.000 \\
\hline
\end{tabular}

Fonte: A. Fornasin, Quanti soldati italiani morirono in prigionia nella Prima guerra mondiale?, in Contemporanea, XXI, 2018.
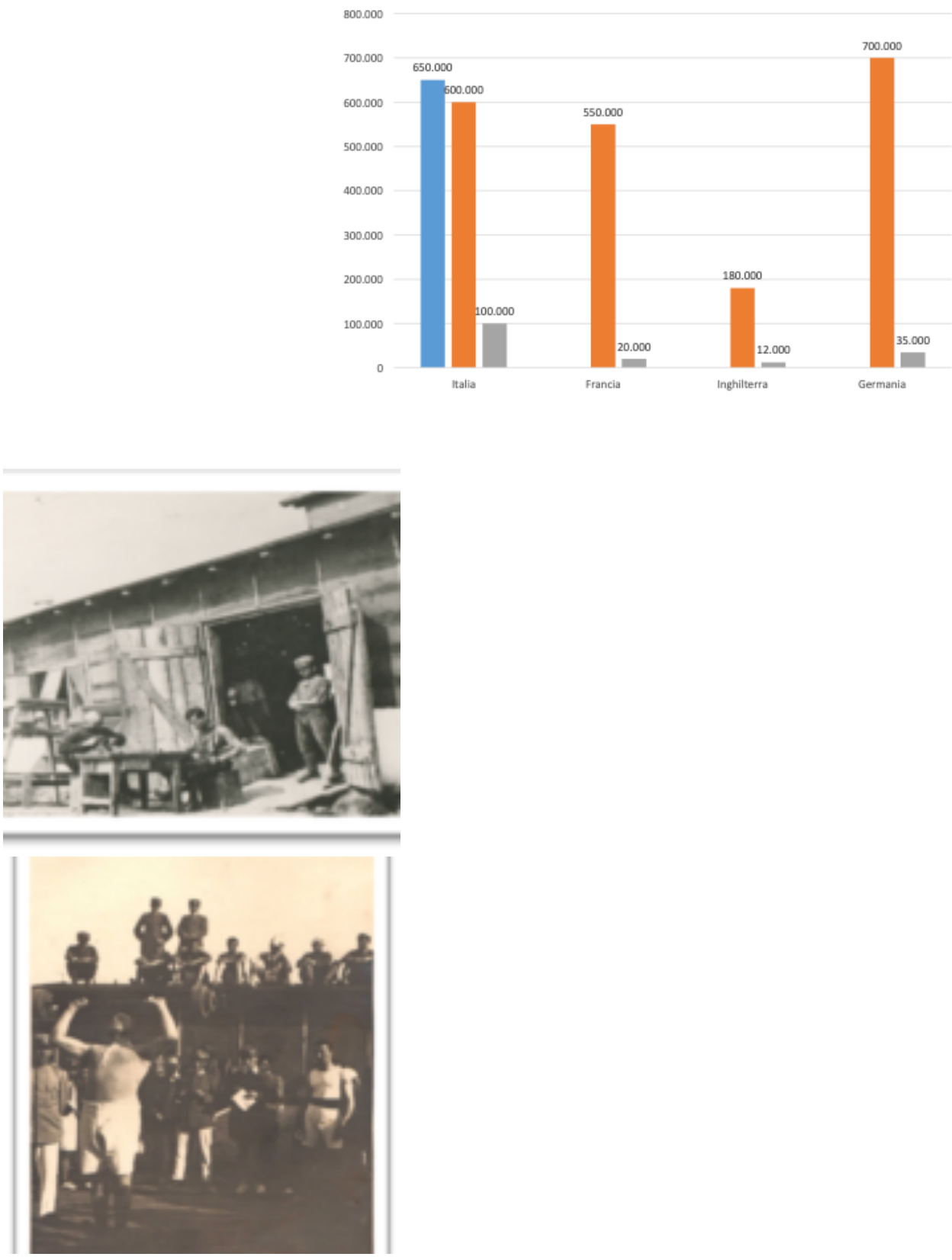

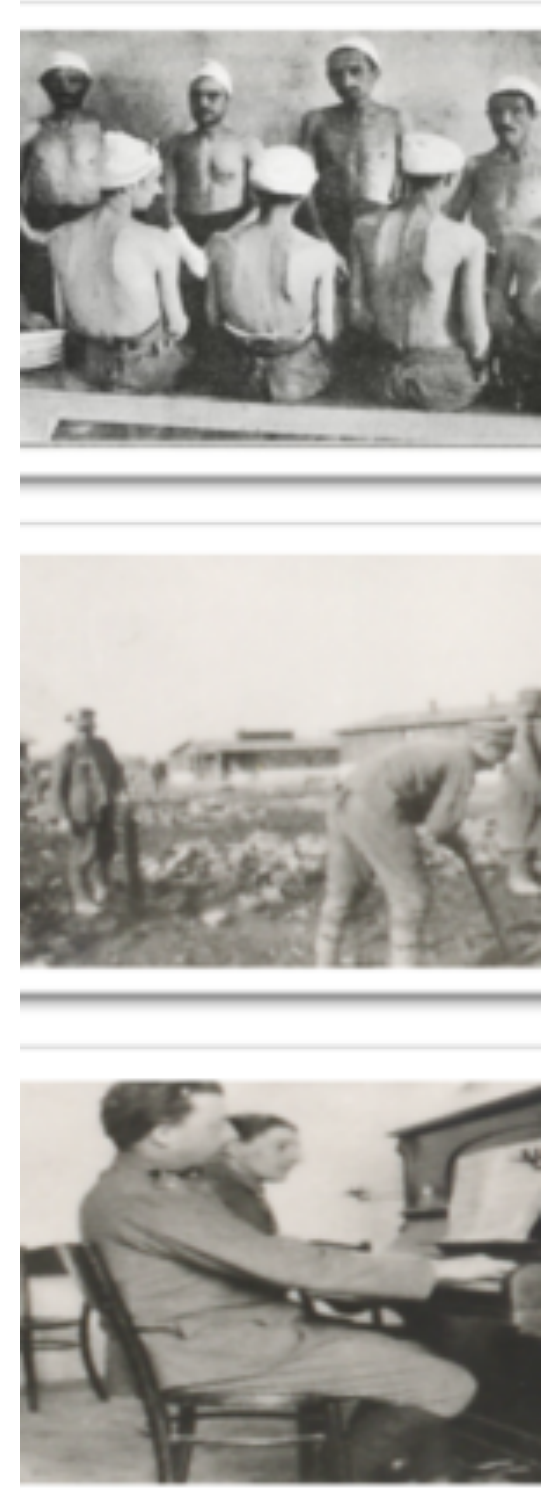

L'ultima slide della lezione, contenente delle parole chiave, è stata funzionale a sintetizzare le varie questioni affrontate e preparare il terreno ad una successiva discussione.

\section{PAROLE CHIAVE}

- Prigionia di massa

- Fronte interno

- Propaganda

- Convenzioni internazionali

- Comunità militare e multietnica 
La lezione frontale sembra rimanere ancora abbastanza radicata all'interno del panorama didattico nazionale, seppur in un contesto di evidente variabilità. Una sua più radicale "rivisitazione", con un approccio maggiormente socio-culturale, come si è cercato di dimostrare, potrebbe valorizzare meglio l'intenso lavoro che i docenti svolgono nel prepararla. Si tratta ovviamente di consigli, stimoli, pratiche e metodologie, che vanno calibrate e armonizzate in base ai singoli contesti scolastici e gruppi classe, ma potenzialmente in grado di favorire un'azione didattica più efficace.

In questo contesto le attività laboratoriali possono diventare una risorsa notevole di stimoli per diversificare la lezione e renderla, più curiosa e accattivante per gli studenti. Le fasi laboratoriali sono momenti utili per proporre consegne e riflessioni teoriche volte a favorire l'autonomia del lavoro nel gruppo classe: "la competenza delle competenze". Tutte le tappe della lezione mirano inoltre a trasformare le conoscenze in competenze agite, far comprendere al gruppo classe come la storia sia utile alla vita di ogni giorno e al proprio futuro, che va oltre la scuola, ma anche a valorizzare il territorio in cui si vive e opera, aprendosi però al globale, alla sua complessità e problematicità. Far dialogare il presente con il passato in questo percorso può facilitare l'approfondimento di temi relativi all'educazione civica, pur partendo da un argomento, il primo conflitto mondiale, che un tempo era la "guerra dei nonni" e oggi ha fatto un salto generazionale più ampio.

Si tratta di pratiche didattiche che nella particolare congiuntura storica di distanziamento sociale, di nuova organizzazione e pianificazione della didattica, possono offrire stimoli e idee: invitano alla multidisciplinarietà, valorizzano il lavoro progettuale e lo studio a casa e le attività asincrone in caso di didattica a distanza, concedono la possibilità di svolgere lezioni oltre le aule scolastiche. L'attuale emergenza sanitaria paradossalmente ci ricorda l'importanza di fare rete sul territorio, non per rimanere rinchiusi dentro i confini del proprio comune, ma per potenziare una scuola che diventi comunità di apprendimento. La didattica sembra essere un buon canale per far collaborare famiglie, enti, istituzioni e associazioni ad un progetto culturale che implementi negli studenti lo spirito di imprenditorialità, la cittadinanza attiva, la resilienza e la loro apertura al mondo.

\section{Bibliografia}

- S. Adorno, Pensare la didattica della storia, in Pensare storicamente. Didattica, laboratori e manuali, Franco Angeli, Milano, 2020,

- P. Battifora, Ė successo un Sessantotto, in Novecento.org, n. 10, agosto 2018

- P. Bernardi (a cura di), Insegnare storia. Guida alla didattica del laboratorio storico, UTET, Torino, 2006

- E. Bricchetto, Fare storia con gli EAS. A lezione del Mediterraneo. Nella scuola secondaria di secondo grado, ELS La Scuola, Brescia, 2016

- A. Brusa, Il laboratorio storico, La Nuova Italia, Firenze, 1991

- A. Brusa suggerisce, Laboratorio del tempo presente, cfr. Parigi, a mente (un po ' più) fredda, in «Novecento.org», 5, dicembre 2015

- P. Burke, Testimoni oculari. Il significato storico delle immagini, Carocci, Roma, 2002

- G. De Luna, L'occhio e l'orecchio dello storico. Le fonti audiovisive nella ricerca e nella didattica della storia, La Nuova Italia, Firenze, 1993

- A. Fossa, G. Nicoletti, E. Peatini, Laboratori per fare storia. Guida pratica alla metodologia della ricerca storico-didattica, Canova, Treviso, 2005

- H. Girardet, Vedere, Toccare, Ascoltare. L'insegnamento della storia attraverso le fonti, Carocci, Roma, 2004 
- F. Hartog, Regimi di storicità. Presentismo e esperienze del tempo, Sellerio, Palermo, 2007; S. Vitali, Lo specchio infranto, in «Storica», 49, 2011

- C. Heimberg, Le questioni socialmente vive e l'apprendimento della storia, in «Mundus online», 1, 2008

- E. Rosso, Le fonti, dalla storiografia alla didattica, in P. Bernardi e F. Monducci, a cura di, Insegnare storia. Guida alla didattica del laboratorio storico, UTET, Torino, 2013

- S. Wineburg, Historical Thinking and Other Unnatural Acts. Charting the Future of Teaching the Past, Temple University Press, Philadelphia, 2001

Note:

[1] Sul laboratorio di storia sempre utili A. Brusa, Il laboratorio storico, La Nuova Italia, Firenze, 1991; P. Bernardi (a cura di), Insegnare storia. Guida alla didattica del laboratorio storico, UTET, Torino, 2006, in particolare pp. 105-132; G. Di Caro, La storia in laboratorio, Carocci, Roma, 2005; A. Fossa, G. Nicoletti, E. Peatini, Laboratori per fare storia. Guida pratica alla metodologia della ricerca storico-didattica, Canova, Treviso, 2005.

[2] Per maggiori informazioni cfr. http://www.sissco.it/articoli/lezioni-di-storia-tecniche-edesperienze-di-didattica-frontale- $2 /$.

[3] C. Heimberg, Le questioni socialmente vive e l'apprendimento della storia, in «Mundus online», 1, 2008; Antonio Brusa suggerisce, ad esempio, il Laboratorio del tempo presente, cfr. Parigi, a mente (un po' più) fredda, in «Novecento.org», 5, dicembre 2015. Sulla didattica controversiale cfr. A. Brusa, A. De Bernardi, S. Guarracino, Storia del Mondo, 3 voll., Laboratori didattici, B. Mondadori, Milano, 2000-2004; P. Battifora, È successo un Sessantotto, in Novecento.org, n. 10, agosto 2018 http://www.novecento.org/didattica-in-classe/e-successo-un-68-laboratorio-dididatticacontroversiale-3093.

[4] S. Adorno, Pensare la didattica della storia, in Pensare storicamente. Didattica, laboratori e manuali, Franco Angeli, Milano, 2020, p. 16. Cfr. anche gli orientamenti in il Manifesto per la Didattica della Storia, a cura del Centro Internazionale di Didattica della Storia e del Patrimonio, Alma Mater Studiorum Università di Bologna, Bologna, 2019, p. 3.; I. Mattozzi, Pensare la nuova storia..., cit., p. 804.

[5] F. Hartog, Regimi di storicità. Presentismo e esperienze del tempo, Sellerio, Palermo, 2007; S. Vitali, Lo specchio infranto, in «Storica», 49, 2011, pp. 63-94.

[6] Su questi temi S. Wineburg, Historical Thinking and Other Unnatural Acts. Charting the Future of Teaching the Past, Temple University Press, Philadelphia, 2001. Dello stesso autore Why Learn History (When it's Alredy on Your Phone), University of Chicago press, Chicago and London, 2018. Per la sua ricezione in Italia P. Ceccoli, Pensare come uno storico non è naturale. Sam Wineburg e le competenze nello studio della storia, in Novecento.org, 11, 2019.

[7] Qualche richiamo sui rischi che si possono verificare: F. Fiore, Memoria, anche E. Bricchetto e A. Perissinotto, Narrazione, sono entrambi in P.C. Rivoltella, E. Bricchetto, A. Perissinotto, a cura di, Media, storia e cittadinanza, La Scuola, Brescia, 2012, rispettivamente alle pagine 41-54 e 5580. Per altri approfondimenti D. Bidussa, C. Greppi, P. Rumiz, Il passato al presente. Raccontare 
la storia oggi, Quaderno Fondazione Gian Giacomo Feltrinelli, 2016, edizione digitale; M. Bloch, Apologia della storia o Mestiere di storico, Einaudi, Torino, 1998 (ed. originale 1949).

[8] E. Bricchetto, Fare storia con gli EAS. A lezione del Mediterraneo. Nella scuola secondaria di secondo grado, ELS La Scuola, Brescia, 2016, p. 29.

[9] P. Burke, Testimoni oculari. Il significato storico delle immagini, Carocci, Roma, 2002. Per qualche esempio D. Chong, La bambina della fotografia. La storia di Kim Phuc e la guerra del Vietnam, Codice, Torino, 2004.

[10] Per un essenziale quadro teorico di riferimento rimando a G. De Luna, L'occhio e l'orecchio dello storico. Le fonti audiovisive nella ricerca e nella didattica della storia, La Nuova Italia, Firenze, 1993; G. De Luna, La passione e la ragione. Fonti e metodi dello storico contemporaneo, La Nuova Italia, Milano, 2001; P. Corrao, P. Viola, Introduzione agli studi di storia, Donzelli, Roma, 2005.

[11] Utili le riflessioni di E. Morin, I sette saperi necessari all'educazione del futuro, Raffaello Cortina Editore, Milano, 2001.

[12] Ma tutto questo implica anche una disponibilità di tempo che spesso i docenti non hanno.

[13] H. Girardet, Vedere, Toccare, Ascoltare. L'insegnamento della storia attraverso le fonti, Carocci, Roma, 2004.

[14] E. Rosso, Le fonti, dalla storiografia alla didattica, in P. Bernardi e F. Monducci, a cura di, Insegnare storia. Guida alla didattica del laboratorio storico, UTET, Torino, 2013, pp. 113-155; E. Musci, Fonti e multimedia per l'insegnamento della storia, in Metodi e strumenti per l'insegnamento e l'apprendimento della storia, in "Quaderni e strumenti per l'insegnamento e l'apprendimento della storia», Edises, Napoli, 2014, pp. 201-253. 